\title{
Characterization and prediction of magnetic losses in Soft Magnetic Composites under distorted induction waveform
}

\author{
O. de la Barrière ${ }^{1}$, C. Appino ${ }^{1}$, F. Fiorillo ${ }^{1}$, C. Ragusa ${ }^{2}$, M. Lecrivain $^{3}$, L. Rocchino ${ }^{1}$, H. Ben Ahmed ${ }^{3}$, M. Gabsi ${ }^{3}$, \\ F. Mazaleyrat ${ }^{3}$ and M. LoBue ${ }^{3}$ \\ ${ }^{1}$ Istituto Nazionale di Ricerca Metrologica (INRIM), Torino, Italy \\ ${ }^{2}$ Dipartimento di Ingegneria Elettrica, Politecnico di Torino, C.so Duca degli Abruzzi 24, 10129 Torino, Italy \\ ${ }^{3}$ SATIE, ENS Cachan, CNRS, UniverSud, 61 av du President Wilson, F-94230 Cachan, France
}

\begin{abstract}
A general approach to magnetic losses in Soft Magnetic Composites (SMC) is discussed. It is applied to exciting conditions typical of applications, where the induction waveform is most frequently non-sinusoidal. The standard predicting approach, based on the Statistical Theory of Losses (STL) and the related concept of loss separation, is extended to the case of granular heterogeneous materials. As a starting point, the classical loss component is calculated, taking into account that in small enough samples the measured loss is independent of the specimen cross-sectional area, because of negligible grain-to-grain (macroscopic) eddy currents. The loss separation is then performed under the conventional sinusoidal induction regime and the STL is used to identify a limited set of parameters, associated with the prediction of the excess loss, by which the energy loss under distorted induction can be predicted. Significant predicting examples are provided, regarding two different SMCs, where the induction waveform is either triangular or distorted by introduction of a third harmonic component.
\end{abstract}

Index Terms-Soft Magnetic Composites, Statistical Theory of Losses, Classical losses, Excess losses, Distorted induction

\section{INTRODUCTION}

$\mathrm{S}^{-1}$ OFT MAGNETIC COMPOSITES (SMCs) display properties of great interest for modern electrical engineering applications. The significant resistivity associated with their heterogeneous granular structure tends to inhibit macroscopic eddy current patterns in magnetic cores and the related energy dissipation [1], making them suitable for applications in highspeed machines [2], and in power electronics. Another advantage derives from the quasi-isotropic behavior of these materials, allowing flexibility in core design [3]. However complications appear in loss analysis, because one has to deal with complex flux regimes, ubiquitously arising in electrical machines and in power electronics [4][5][6], while the data provided by the manufacturers typically refer, according to the standards, to sinusoidal induction. A loss model, simple enough to ensure acceptable computation time, quantitatively accounting for the role of flux distortions, would be therefore important for the accurate prediction of iron losses.

In a previous paper, we have addressed the general problem of experimentally assessing and theoretical predicting the magnetic losses in SMCs [7] by means of measurements carried out under sinusoidal induction up to $10 \mathrm{kHz}$, and their interpretation in the framework of the Statistical Theory of Losses (STL) [8]. With respect to the soft laminations, peculiar differences have emerged in the statistics of the Magnetic Objects (MOs), which are the regions reversing the magnetization in any sample cross-section in a correlated fashion. In particular, it has been shown that the number $n_{\mathrm{o}}$ of MOs simultaneously active under quasi-static excitation is far from unity, contrary to what generally happens in laminated materials [8]. It is consequently observed that the excess loss component $W_{\text {exc }}$ attains the typical $W_{\text {exc }} \propto f^{1 / 2}$ dependence only when the magnetizing frequency $f$ approaches the $\mathrm{kHz}$ range [7]. This engenders complications in the treatment of the losses under distorted induction waveform, which have been discussed and predicted in soft laminations relying on such a $W_{\text {exc }} \propto f^{1 / 2}$ rule [9][10][11].

A rate-dependent hysteresis model for magnetic losses under non-sinusoidal induction in SMCs has been recently proposed [12]. This approach, however, disregards the classical losses and is restricted to a maximum frequency of a few hundred $\mathrm{Hz}$, where SMCs hardly exhibit any dynamic loss contribution.

As a first step in the development of a loss model in SMCs, we need to establish what is the scale to be considered for the involved eddy currents (e.c.). That is, we wish to understand whether the currents remain mostly confined within the particles (in which case we define them as microscopic e.c.) or they can appreciably flow upon the scale of the sample cross-section (macroscopic e.c.) through random interparticle contacts. To this end, the loss is measured on ring samples of commercial SMCs having increasing crosssectional area $S_{0}$. It is concluded that macroscopic e.c. do not affect the loss with sufficiently small $S_{\mathrm{o}}$ values (i.e. with $S_{\mathrm{o}}=$ $5 \mathrm{~mm} \times 5 \mathrm{~mm}$ or lower). This brings about a good simplification in our analysis, which will be focused here in the prediction of the role of the microscopic e.c. We shall, in particular, start with the calculation for sinusoidal induction of the classical losses pertaining to the individual particles. In this specific context we qualitatively attach a microscopic character to the e.c. flowing on the scale of the particle. Based then on micrographic inspections, the sample cross-section is assumed to be covered by rectangular grains with suitably distributed dimensions. Different regular shapes (such as squares or ellipses) could equivalently be assumed, with little effect on the final calculated loss figure.

With the so obtained classical losses and the hysteresis loss component secured by extrapolation of the measured loss to the frequency $f \rightarrow 0$, the residual excess loss is interpreted in the framework of the STL, the parameters of the model being 
identified with the measurements made under sinusoidal induction. The results are then used to predict the magnetic losses under non-sinusoidal induction. This approach is validated by experiments performed in a broad frequency range (up to $8 \mathrm{kHz}$ for the fundamental harmonic), as potentially required for applications in very high speed electrical machines. The complex problems involved with local minima of the induction (minor loops) require quasistatic loop modeling and will be treated in a forthcoming paper.

The paper is organized as follows:

- in Section II, the problems related to the classical loss computation are dealt with.

- in Section III, the parameters identification, carried out for sinusoidal induction, is discussed.

- in Section IV the loss prediction is compared with experiments performed, for a range of peak induction values, either with triangular induction or distortion introduced by a third harmonic component.

- $\quad$ in Section $\mathrm{V}$, the conclusions of the work are given.

\section{THE CLASSICAL LOSS COMPONENT AND ITS CALCULATION}

The concept of classical loss, defined as the energy dissipated in a magnet fictitiously deprived of the magnetic domains and, as such, representing a lower limiting value for the loss, has been given clear physical meaning in the statistical treatment of the magnetization process in laminated materials [8]. According to the STL, the classical loss $W_{\mathrm{cl}}$ adds to the hysteresis (DC) $W_{\text {hyst }}$ and excess loss $W_{\text {exc }}$ components to provide the total loss $W$. Calculating $W_{\mathrm{cl}}$ in heterogeneous materials is a more complicated matter, because, as previously stated, eddy current patterns can circulate at the same time on the scale of the individual particles (microscopic) and that of the sample cross-section (macroscopic) [13][14][15][16]. The problem is sometimes addressed from a phenomenological viewpoint, assuming that $W_{\mathrm{cl}} \propto f$ and $W_{\mathrm{exc}} \propto f^{1 / 2}$ and determining the proportionality constants by best fit of the measured loss over a range of frequencies [17]. This is an oversimplification, as the experiments show that the excess loss in SMCs follows a more complex frequency dependence than $W_{\text {exc }} \propto f^{1 / 2}$ [7]. Whereas the microscopic e.c. contribution it is often studied assuming spheroidal particles [18], a few authors consider the macroscopic e.c. and compute the classical loss by a Finite Element Method (FEM), solving a diffusion equation upon the whole sample [19][20]. This procedure exploits the so-called "ohmic" resistivity measured under DC conditions. This approach may be straightforward in mildly compacted materials [13], whose resistivity is mainly due to imperfections of the intergrain insulator, but its application to strongly compacted materials, where the electrical resistivity is due to intergrain percolation contacts, is dubious [16].

In this work we have experimentally found that the magnetic losses can increase with the sample size (i.e. crosssectional area) only beyond a certain critical dimension. With sufficiently small samples, the macroscopic e.c. play a negligible role. We shall focus here on such cases, in order to calculate the contribution of the microscopic e.c.

Two types of commercial SMCs have been investigated: one developed for electrical machines (Höganäs Prototyping, material A), the other for power electronics (Höganäs 110i1P, material B) [21]. Both materials are made by compacting pure iron particles. Their main physical properties, as reported by the manufacturer, are shown in Table I. The resistivity of the composite is remarkably 3 orders of magnitude compared to pure $\mathrm{Fe}\left(\rho_{\mathrm{Fe}}=104 \cdot 10^{-9} \Omega \cdot \mathrm{m}\right.$ at room temperature $)$.

TABLE I

PHYSICAL PARAMETERS OF THE MATERIALS (FROM THE MANUFACTURER [21])

\begin{tabular}{llll}
\hline \hline & & Material A & Material B \\
Relative permeability & $\mu_{\mathrm{r}}$ & 450 & 110 \\
Electrical resistivity & $\rho(\Omega \cdot \mathrm{m})$ & $280 \cdot 10^{-6}$ & $7600 \cdot 10^{-6}$ \\
Density & $\delta\left(\mathrm{kg} / \mathrm{m}^{3}\right)$ & 7450 & 7260 \\
\hline
\end{tabular}

Magnetic testing has been performed as a function of frequency in ring shaped SMC samples by means of hysteresisgraph-wattmeter with digital control of the induction waveform. The setup is schematically shown in Fig. 1. The specific loss $W$ is obtained by computing the area of the hysteresis cycle. The power source is a DC-20 kHz $5 \mathrm{kVA}$ linear amplifier (CROWN mod. 5000VZ). When dealing with large samples (3, and 4 in Table II), the required apparent power is so high that it can be necessary to add a capacitor $C$ in series with the primary winding, in order to overcome the amplifier voltage limit. Due to the low thermal conductivity of SMCs, sample heating can soon become a problem with the increase of frequency and peak polarization value $J_{\mathrm{p}}$. For this reason, the test specimen is contained in a thermal bath at $0^{\circ} \mathrm{C}$ and the measuring time is $1 \mathrm{~s}$ at most.

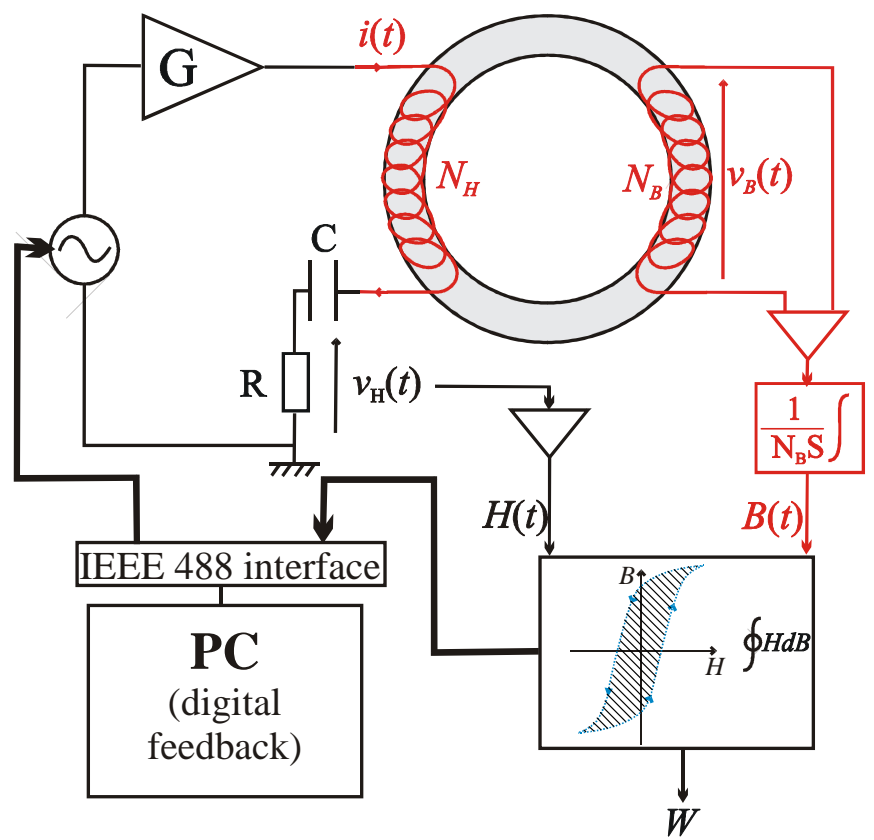

Fig. 1 - Schematic view of the employed hysteresisgraph-wattmeter.

\section{A) Macroscopic eddy currents.}

The concept of eddy current patterns arising upon two different scales, that of the individual iron particle (microscopic e.c.) and that of the sample cross-section (macroscopic e.c.) leads us to 
define the classical loss as the sum of microscopic macroscopic terms:

$$
W_{\text {class }}=W_{\text {class,MAC }}+W_{\text {class,MIC }}
$$

It is easily demonstrated that this is the case under the reasonable condition that the macroscopic e.c. have constant value over a distance spanning the particle size. It is also obviously concluded that only the term $W_{\text {class, MAC in (1) }}$ should depend on the cross-sectional area of the sample. To clarify this matter, we have measured the energy loss $W(f)$ on toroidal samples of different cross-sectional areas (material A), ranging from $2.5 \mathrm{~mm} \times 2.5 \mathrm{~mm}$ to $20 \mathrm{~mm} \times 10 \mathrm{~mm}$ (Table II). The ratio of outside to inside diameter was always taken around 1.1, to ensure good homogenetity of the induction throughout the sample cross-section.

TABLE II

THE INVESTIGATED SPECIMENS (MATERIAL A)

\begin{tabular}{cc}
\hline \hline Ring sample & Cross-section $(\mathrm{mm} \times \mathrm{mm})$ \\
\hline 1 & $2.5 \times 2.5$ \\
2 & $5 \times 5$ \\
3 & $10 \times 10$ \\
4 & $20 \times 10$ \\
\hline
\end{tabular}

The magnetic losses were measured under controlled sinusoidal induction in all these samples for the two peak polarization values $J_{\mathrm{p}}=0.5 \mathrm{~T}$ and $J_{\mathrm{p}}=1 \mathrm{~T}$ in the frequency ranges DC-10 kHz (toroids 1 and 2) and $\mathrm{DC}-2 \mathrm{kHz}$ (toroids 3 and 4). The $2 \mathrm{kHz}$ upper limit in the bigger samples is imposed by the maximum available exciting power.

It is apparent in Fig. 2 that $W(f)$ increases faster with $f$ in the bigger samples 3 and 4, a clear effect of macroscopic e.c. rising with cross-sectional area. But no significant differences can be found in the toroids 1 and 2, even at the highest frequencies. As discussed in [16], macroscopic eddy current loops may form, because of the presence of random contacts among grains formed during the compaction process. The loop size depends on the number of contacts, that is, on the compaction pressure. In sufficiently small samples no closed macroscopic random paths can form, the e.c. are confined to the scale of the particle, and $W_{\text {class }}=W_{\text {class,MIC. The }}$ contribution $\mathrm{W}_{\text {class,MAC }}$ will appear as soon as the critical sample size is achieved. According to the results of Fig. 2 (material A), this size appears intermediate between that of toroid 2 and toroid 3. However, the computation of these macroscopic eddy currents is by no mean obvious, because it requires a specific model for the intergrain random contacts and the related paths. For the time being, we shall focus on the properties of samples small enough (e.g. samples 1 and 2) to make the macroscopic eddy currents negligible.

B. Microscopic eddy currents.

The grain structure of the investigated SMCs has been observed using a scanning electron microscope. Fig. 3 provides an example of micrograph taken of material A (for the material B an example is shown in [7]). It is observed that the particles in the material $\mathrm{A}$ are bigger and have undergone more severe plastic deformation than in the material B, andesulting in density closer to that of iron $\left(\delta_{\mathrm{A}}=7450 \mathrm{~kg} \cdot \mathrm{m}^{-3} ; \delta_{\mathrm{B}}\right.$ $\left.=7260 \mathrm{~kg} \cdot \mathrm{m}^{-3} ; \delta_{\mathrm{Fe}}=7870 \mathrm{~kg} \cdot \mathrm{m}^{-3}\right)$.
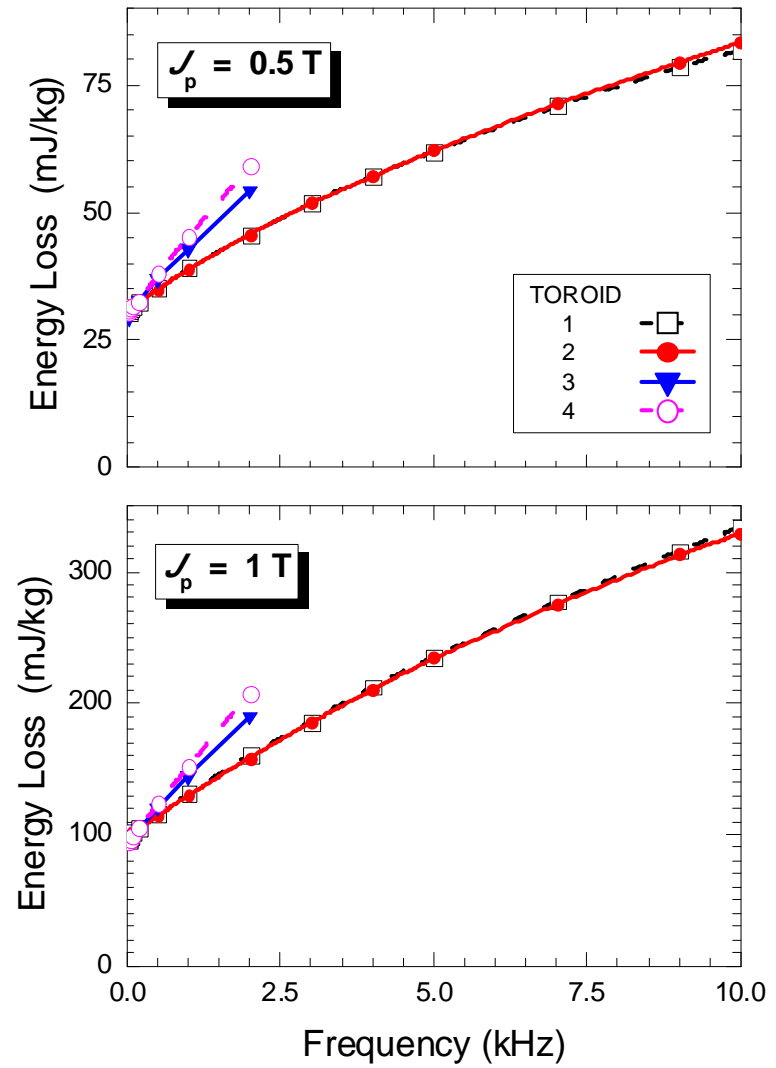

Fig. 2 - Energy loss $W$ versus frequency $f$ in toroids of material A having different cross-sectional area. Peak polarization values $J_{\mathrm{p}}=0.5 \mathrm{~T}$ and $J_{\mathrm{p}}=1 \mathrm{~T}$. A faster increase of $W$ versus $f$ is observed in the bigger toroids 3 and 4 .

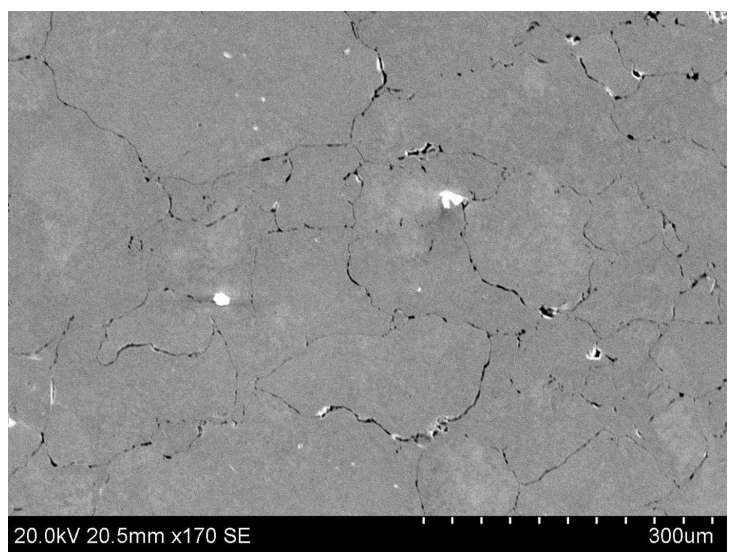

Fig. 3 - Cross-sectional micrographic view of a type A SMC sample.

In order to calculate $W_{\text {class,MIC }}$, the classical eddy current loss associated with the currents circulating within the particles, we assume the sample cross-section covered with rectangular grains, accounting for the shape of the actual irregularly shaped particles. This choice is justified by the fact that circular grains, frequently invoked in the literature [13][14], cannot fill the plane and take the non equiax nature of most particles into account. Here we first treat the case of the 
individual particle with rectangular cross-section, to further generalize the problem to an ensemble of particles with distributed geometrical parameters.

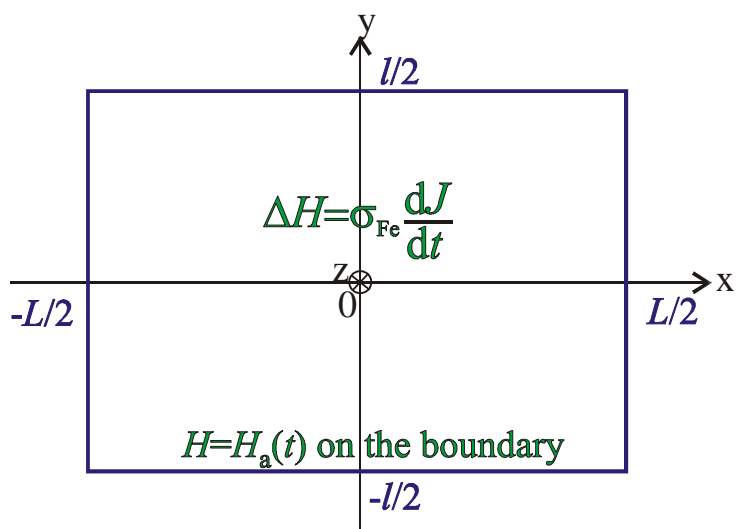

Fig. 4 - Cross-section of the model rectangular iron particle and Poisson's equation for the magnetic field under the time dependent applied field $H_{\mathrm{a}}(t)$.

\section{1) Eddy currents in the equivalent rectangular particle}

An equivalent rectangular particle of aspect ratio $R=l / L$ and cross-sectional area $S=L \cdot l$, belonging to the $(x, y)$ plane (Fig. 4) is subjected to the $z$-directed time-varying applied field $\boldsymbol{H}_{\mathrm{a}}(t)$ and the ensuing polarization $\boldsymbol{J}(t)$ (taken equal to the induction $\boldsymbol{B}(t))$. Negligible skin effect is envisaged. This assumption can be verified by computing, for a frequency $f=10000 \mathrm{~Hz}$ (equal to the maximal test frequency), the skin dept (s.d.) thanks to the classical formula:

$$
\text { s.d. }=\frac{1}{\sqrt{\sigma_{\mathrm{Fe}} \mu_{\mathrm{r}} \mu_{0} \pi f}}
$$

Where $\sigma_{\mathrm{Fe}}=1 / \rho_{\mathrm{Fe}}=9.93 \cdot 10^{6} \mathrm{~S} \cdot \mathrm{m}^{-1}$ is the conductivity of pure iron, $\mu_{\mathrm{r}} \approx 400$ is an estimation of the composite relative magnetic permeability given by the manufacturer [21]. The skin dept found by this formula is $80 \mu \mathrm{m}$, which is superior to the half of the mean grain dimension of material $\mathrm{A}\langle s\rangle_{\mathrm{A}}=$ $114 \mu \mathrm{m}$ (assuming the grains as squares).

The 2-D Poisson's equation for the magnetic field $\Delta \boldsymbol{H}=\sigma_{\mathrm{Fe}} \cdot d \boldsymbol{J} / d t$, where $\boldsymbol{H}$ is the sum of applied and eddy current fields, and $\boldsymbol{H}(t)=\boldsymbol{H}_{\mathrm{a}}(t)$ on the particle boundary, is solved by finite element analysis and the 2-D eddy current density $j(x, y)$ in each point of the grain is calculated. Note that the stated boundary condition $\boldsymbol{H}(t)=\boldsymbol{H}_{\mathrm{a}}(t)$ is the same on the particle contour and the sample surface. This is acceptable in the absence of macroscopic eddy currents [22]. By integrating | $\left.\boldsymbol{j}(x, y)\right|^{2}$ over the grain area, the energy loss is obtained as

$$
W_{\text {class,MIC }}^{(R, S)}\left(J_{\mathrm{p}}, f\right)=\frac{1}{\rho_{\mathrm{Fe}}} \cdot K(R) \cdot S\left\langle\dot{J}^{2}(t)\right\rangle \quad, \quad\left[\mathrm{J} / \mathrm{m}^{3}\right]
$$

where $T=1 / f$ and the role of the particle aspect ratio is included in the dimensionless function $K(R)$. The calculated behavior of $K(R)$, shown in Fig. 5, puts in evidence that for given $J(t)$ and $S$, the loss is reduced if the width-to-length ratio is small (a "flat" particle).

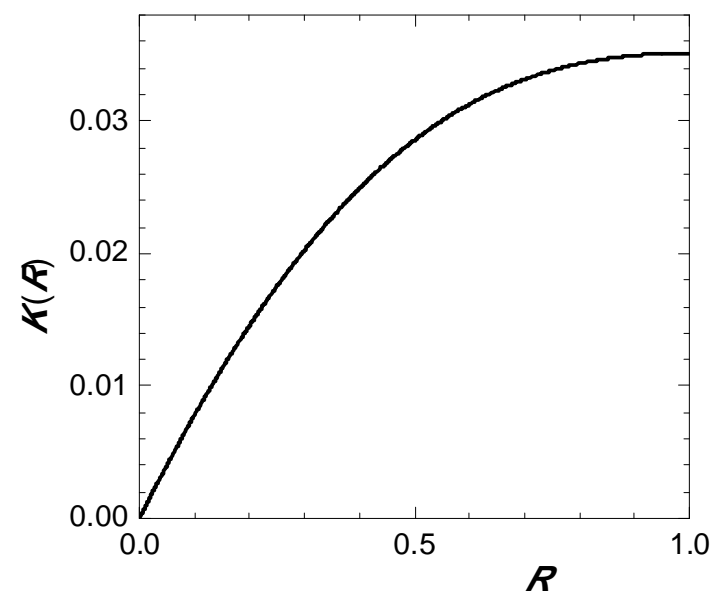

Fig. 5: Function $K(R)$ describing the evolution of the classical loss with the aspect ratio $R=l / L$ in the equivalent rectangular particle.

The formula (2) permits to retrieve the well-known expression of the classical loss in a lamination in the limit $l / L \rightarrow 0$. This is explained in the appendix.

2) Statistical distribution of the particle size.

Micrographic investigation can provide average values for $S$ and $R$ and the rectangular grain model can accordingly be adopted, then the previous calculation is applied, leading to a figure for $W_{\text {class,MIC }}$ [7]. Fig. 6 illustrates the relationship between the real irregular grain and the model one. But, given the non-linear nature of the problem, a refined approach taking the dispersion of $S$ and $R$ values into account would lead to a more realistic prediction for the microscopic classical loss by integrating $W_{\text {class,MIC }}(S, R)$ over the distribution function $f(S$, $R)$.

An extensive micrographic analysis centered on the use of an image processing software allows to find experimental

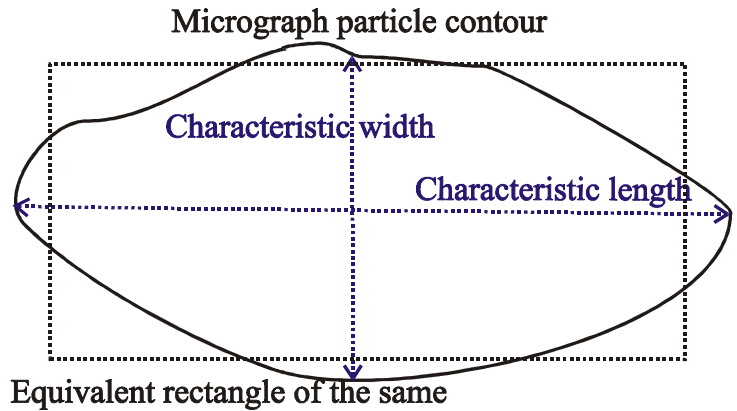
surface and proportions

Fig. 6: Characteristic dimensions of a real irregular particle and the equivalent rectangular grain having same area $S$ and aspect ratio $R$, defined as the ratio between the characteristic width and the characteristic length.

distribution functions for $S$ and $R$ in both materials. $S$ and $R$ are non-independent random variables. They appear to follow a bi-variate lognormal law: 


$$
\begin{aligned}
f(S, R) & =\frac{1}{S \cdot R} \frac{1}{2 \pi \xi_{S} \xi_{R} \sqrt{1-\chi_{S, R}}} \exp \left\{\frac{\left(\ln S-\alpha_{S}\right)^{2}}{\xi_{S}}\right. \\
& \left.+\frac{\left(\ln R-\alpha_{R}\right)^{2}}{\xi_{R}}-\frac{2 \chi_{S, R}\left(\ln S-\alpha_{S}\right)\left(\ln R-\alpha_{R}\right)}{\xi_{S} \xi_{R}}\right\}
\end{aligned}
$$

The parameters appearing in (4) can be estimated by statistical methods [23], and their definitions and values, for both materials, are summarized in Table III.

TABLE III

\begin{tabular}{|c|c|c|c|}
\hline Parameter & Definition & Material A & Material B \\
\hline$\alpha_{S}$ & $\begin{array}{l}\text { Mean value of the particle cross- } \\
\text { sectional area logarithm }\end{array}$ & -18.56 & -21.4 \\
\hline$\alpha_{R}$ & $\begin{array}{l}\text { Mean value of the particle aspect } \\
\text { ratio logarithm }\end{array}$ & -0.45 & -0.50 \\
\hline$\xi_{S}$ & $\begin{array}{l}\text { Standard deviation of the particle } \\
\text { cross-sectional area logarithm }\end{array}$ & 0.92 & 0.46 \\
\hline$\xi_{R}$ & $\begin{array}{l}\text { Standard deviation of the particle } \\
\text { aspect ratio logarithm }\end{array}$ & 0.32 & 0.16 \\
\hline$\chi_{S, R}$ & $\begin{array}{l}\text { Correlation coefficient between } \ln (S) \\
\text { and } \ln (R)\end{array}$ & -0.27 & -0.52 \\
\hline
\end{tabular}

PARAMETERS OF THE LOG-NORMAL DISTRIBUTION FUNCTION (4)

The mean size of an equivalent square particle is $\langle s\rangle_{\mathrm{A}}=$ $114 \mu \mathrm{m}$ and $\langle s\rangle_{\mathrm{B}}=29.5 \mu \mathrm{m}$ for the materials $\mathrm{A}$ and $\mathrm{B}$, respectively. Using this statistical model, the microscopic loss in the SMC can be computed according to the equation

$$
\begin{aligned}
& W_{\mathrm{class}, \mathrm{MIC}}^{(R, S)}\left(J_{\mathrm{p}}, f\right)=\frac{\delta}{\delta_{\mathrm{Fe}}} \cdot \frac{1}{\rho_{\mathrm{Fe}}} \cdot K(R) S f \int_{0}^{T} \dot{J}^{2}(t) d t . \\
& \iint_{0}^{\infty} \int_{0}^{\infty} f(S, R) K(R) S d R d S
\end{aligned}
$$

The ratio $\left(\delta / \delta_{\mathrm{Fe}}\right)<1$ between SMC and iron densities accounts for the fact that the insulating material does not participate in the generation of the eddy current loss.

We compare in Fig. 7 the prediction of the classical loss made in materials A and B using either (5) or (3), that is considering or not the statistical distribution of $S$ and $R$. While one can appreciate the role of the particle size, with $W_{\text {class,MIC }}$ going roughly as $\langle s\rangle^{2}$, it is noted that for the material $\mathrm{A}$, characterized with quite wider $(R, S)$ distribution than material B, a correspondingly larger effect (about $8 \%$ increase) is predicted.

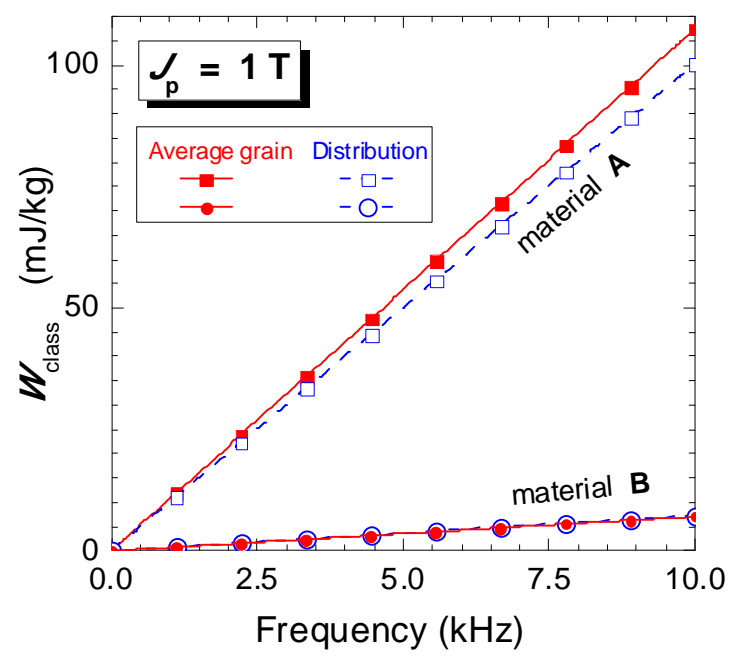

Fig. 7- Microscopic classical loss $W_{\text {class,MIC }}$ predicted in materials $\mathrm{A}$ and $\mathrm{B}$ (sinusoidal polarization, peak value $J_{\mathrm{p}}=1 \mathrm{~T}$ ) either taking all grains equal to the actual average grain (dashed lines) or considering the distribution of crosssectional area $\mathrm{S}$ and aspect ratio $\mathrm{R}$.

\section{3) Rectangular versus elliptical particles.}

The model rectangular particles conveniently fill the available space, consistent with the high density of the SMCs. We may however wonder to what extent the calculated $W_{\text {class,MIC }}$ is affected by the specific shape of the model particle. To this end, we have performed the eddy current calculation for elliptical particles, a representation of the real particles more realistic than the circular/spherical grains conventionally assumed in the literature [13][14]. Again, the computations have been carried out using finite elements, because analytical formulations only exist for circular or spherical shapes [18]. The problem to solve is similar to the one shown in Fig. 4, now with an elliptical boundary. Thus, taking the aspect ratio $R=r_{\mathrm{m}} / r_{\mathrm{M}}$, where $r_{\mathrm{m}}$ and $r_{\mathrm{M}}$ and are the minor and major axis of the ellipse, respectively, and the area $S=\pi r_{\mathrm{m}} r_{\mathrm{M}}$, the Poisson equation for the magnetic field $\Delta \boldsymbol{H}=\sigma_{\mathrm{Fe}} \cdot d \boldsymbol{J} / d t$ is solved using FEM with Dirichlet boundary conditions. The classical eddy current loss $W_{\text {class,MIC }}^{\left(r_{\mathrm{M}}, r_{\mathrm{m}}\right)}\left(J_{\mathrm{p}}, f\right)$ is obtained after integration of the squared current density on the equivalent ellipse area. Using the statistical distribution $f(S, R)$, we obtain

$$
W_{\text {class,MiC }}^{(\text {(ELII) }}\left(J_{\mathrm{p}}, f\right)=\frac{\delta}{\delta_{\mathrm{Fe}}} \int_{0}^{\infty} \int_{0}^{\infty} f(S, R) W_{\text {class,MIC }}^{\left(r_{\mathrm{M}}, r_{\mathrm{m}}\right)}\left(J_{\mathrm{p}}, f\right) d R d S\left[\mathrm{~J} / \mathrm{m}^{3}\right]
$$

Fig. 8 provides a comparison of the loss calculated in the material A (sinusoidal polarization, $J_{\mathrm{p}}=1 \mathrm{~T}$ ) for the rectangular (5) and elliptical (6) model grain. A 10\% higher 


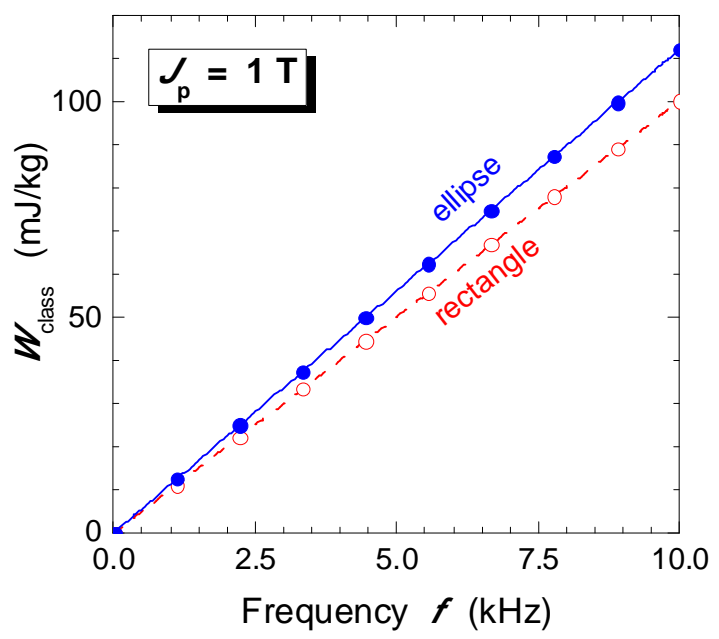

Fig. 8 - Material A. Classical eddy current loss predicted starting from either rectangular or elliptical model particle and distributed geometrical parameters.

loss figure is obtained, for the same $(S, R)$ distribution, with the elliptical particle. We conclude that the true value of $W_{\text {class,MIC }}$ should lie between these two values. In the following calculations, the rectangular model particle will be adopted.

\section{LOSS UNDER SINUSOIDAL POLARIZATION: PARAMETERS IDENTIFICATION}

Having calculated, according to the previous discussion, the classical energy loss component, we can proceed to determine the other two components: the hysteresis loss $W_{\text {hyst }}$ and the excess loss $W_{\text {exc }}$ [8]. We perform this operation for sinusoidal induction waveform.

Hysteresis energy loss $W_{\text {hyst }}$ This component depends, in the absence of minor loops, exclusively on the peak polarization $J_{\mathrm{p}}$ [9]. It is obtained by experiments, namely by extrapolating, at any $J_{\mathrm{p}}$ value, the measured loss versus frequency curve to zero frequency.

Excess energy loss $W_{\text {exc }}$. This term, arising from the inhomogeneous character of the magnetization process (i.e. the domain wall displacements) is experimentally obtained by subtracting at each frequency $\mathrm{W}_{\text {hyst }}$ and $W_{\text {class }}$ from the total loss $W$. It is theoretically predicted, under very general terms, starting from the expression for the instantaneous specific power loss $P_{\text {exc }}(t)$ [10], according to the expression

$$
P_{\text {exc }}(t)=\frac{n_{\mathrm{o}} V_{\mathrm{o}}}{2}\left(\sqrt{1+\frac{4 \sigma G S_{o} V_{\mathrm{o}}}{n_{\mathrm{o}}^{2} V_{\mathrm{o}}^{2}}|\dot{J}(t)|}-1\right)|\dot{J}(t)| \quad\left[\mathrm{W} / \mathrm{m}^{3}\right]
$$

where $n_{\mathrm{o}}$ is the number of MOs under quasi-static excitation, $S_{\mathrm{o}}$ is the cross-sectional area of the sample, $G=0.1356$, and $V_{\mathrm{o}}$ is related to the statistical distribution of the reversal fields for the MOs [8]. The parameters $n_{\mathrm{o}}$ and $V_{\mathrm{o}}$ both depend on $J_{\mathrm{p}}$. $W_{\text {exc }}$, obtained by integrating Eq. (3) over the period $T$, is expressed for sinusoidal $J(t)$ as

$$
\begin{aligned}
& W_{\mathrm{exc}}^{(\mathrm{sin})}\left(J_{\mathrm{p}}, f\right)=2 n_{\mathrm{o}} V_{\mathrm{o}} J_{\mathrm{p}} \\
& \quad \cdot \int_{0}^{\pi / 2}\left(\sqrt{1+\frac{8 \sigma G S_{\mathrm{o}} V_{\mathrm{o}}}{n_{\mathrm{o}}^{2} V_{\mathrm{o}}^{2}} \pi f J_{\mathrm{p}} \cos \varphi}-1\right) \cos \varphi \mathrm{d} \varphi \cdot\left[\mathrm{J} / \mathrm{m}^{3}\right]
\end{aligned}
$$

By fitting the experimental $W_{\text {exc }}^{(\sin )}\left(J_{\mathrm{p}}, f\right)$ it with Eq. (7), $n_{\mathrm{o}}\left(J_{\mathrm{p}}\right)$ and $V_{\mathrm{o}}\left(J_{\mathrm{p}}\right)$ are identified. SMC materials have unique response in terms of the parameter $n_{\mathrm{o}}\left(J_{\mathrm{p}}\right)$, which can be pretty high [7]. By opposition, in magnetic laminations it is a few units at most, permitting one to reduce (7) to the well known square root law $W_{\mathrm{exc}}^{(\mathrm{sin})}=8.76 \sqrt{\sigma G S_{\mathrm{o}} V_{\mathrm{o}}} J_{\mathrm{p}}^{3 / 2} f^{1 / 2}$.

Loss measurements performed in the A-type and B-type SMC ring samples under sinusoidal flux over a wide range of frequencies (from quasi-static excitation up to $10 \mathrm{kHz}$ ) and at $J_{\mathrm{p}}$ values ranging between $0.1 \mathrm{~T}$ and $1.25 \mathrm{~T}$, permit one to identify the quantities $W_{\text {hyst }}\left(J_{\mathrm{p}}\right), n_{\mathrm{o}}\left(J_{\mathrm{p}}\right)$, and $V_{\mathrm{o}}\left(J_{\mathrm{p}}\right)$. Fig. 9a shows the experimental behavior of the hysteresis loss $W_{\text {hyst }}\left(J_{\mathrm{p}}\right)$ in the A-type SMC, showing a power law dependence $W_{\text {hyst }}\left(J_{\mathrm{p}}\right) \propto J_{\mathrm{p}}{ }^{1.7}$. The excess loss parameters $V_{\mathrm{o}}\left(J_{\mathrm{p}}\right)$ and $n_{\mathrm{o}}\left(J_{\mathrm{p}}\right) \cdot V_{\mathrm{o}}\left(J_{\mathrm{p}}\right)$, appearing in (7) and (8) are equally observed to increase with $J_{\mathrm{p}}$ (Fig. 9b) Quite similar behaviors are observed in the B-type SMCs.

\section{LOSS UNDER DISTORTED INDUCTION WAVEFORM}

Based on the previous separation procedure, performed for sinusoidal flux, we can proceed to the calculation of the energy losses under non-sinusoidal polarization. Two different examples of loss calculation are provided in this Section, where $J(t)$ is obtained either by combination of fundamental and third harmonics or as a triangular function.

\section{1) Distortion of $J(t)$ introduced by a third harmonic.}

Let us consider the time dependent polarization

$$
J(t)=J_{1} \sin (\omega t)+J_{3} \sin \left(3 \omega t+\varphi_{3}\right)
$$

where $\omega=2 \pi f$, and the harmonics $J_{1}$ and $J_{3}$ satisfy the conditions $J_{3} / J_{1}=0.1$ with $J_{\mathrm{p}}=$ const, such as a phase shift $\varphi_{3}$ between $0^{\circ}$ and $180^{\circ}$, no minor loop can form for so $W_{\text {hyst }}\left(J_{\mathrm{p}}\right)$ is not affected by distortion of $J(t)$. Examples of $J(t)$ waveforms with $J_{\mathrm{p}}=1 \mathrm{~T}$ and different $\varphi_{3}$ values are shown in Fig. 10. For any given $J_{\mathrm{p}}$, the hysteresis loss $W_{\text {hyst }}\left(J_{\mathrm{p}}\right)($ Fig. 9a) is identical to the one obtained with sinusoidal flux. The component $W_{\text {class }}^{\text {(dist) }}\left(J_{\mathrm{p}}, f\right)$ is immediately obtained, either through (4) or by retrieving and summing up the $W_{\text {class }}$ (sin) values calculated for sinusoidal induction at the frequencies $f$ and $3 f$ and the peak polarizations $J_{1}$ and $J_{3}$, respectively. Because of its quadratic dependence on the induction derivative, $W_{\text {class }}{ }^{\text {(dist) }}$ does not depend on the phase $\varphi_{3}$. The excess loss $W_{\text {exc }}$ (dist) is finally calculated using (7) and introducing the term $\dot{J}(t)$ as the derivative of (9). The parameters $V_{\mathrm{o}}\left(J_{\mathrm{p}}\right)$ and $n_{\mathrm{o}}\left(J_{\mathrm{p}}\right) V_{\mathrm{o}}\left(J_{\mathrm{p}}\right)$ in (7) are retrieved, as discussed in Section III, from the loss measured under sinusoidal $J(t)$ (see Fig. 9b for the type-A material). Fig. 11 shows the evolution with the phase shift $\varphi_{3}\left(0 \leq \varphi_{3} \leq 180^{\circ}\right)$ of the energy loss $W^{(\text {dist })}$ measured under the distorted $J(t)$ given by (8) with $J_{\mathrm{p}}=1 \mathrm{~T}$ and $J_{3} / J_{1}=0.1$ (symbols) and the prediction made according to the above procedure (solid line). 
Two examples, corresponding to the fundamental frequencies $f=2 \mathrm{kHz}$ and $f=7 \mathrm{kHz}$ are given, where the evolution of the loss components with the distortion compared with the loss measured with sinusoidal $J(t)$ is visible. It is noted that the distortion associated with $\varphi_{3}$ values closed to $180^{\circ}$ leads to a decrease of the loss. Fig. 12 shows that the effect of distortion can also be predicted with good accuracy in the type-B composite. This kind of material exhibits strong hysteresis loss component and reduced classical losses, because of its fine granular structure.

\section{2) Triangular polarization waveform}

The above discussed prediction approach can be straightforwardly applied to triangular induction. But, since $\dot{J}(t)$ is a constant, it is simpler to get integrate analytically (7), the expression for the excess loss:

$$
W_{\mathrm{exc}}^{\text {(tri) }}\left(J_{\mathrm{p}}, f\right)=2 n_{\mathrm{o}} V_{\mathrm{o}} J_{\mathrm{p}}\left(\sqrt{1+\frac{16 \sigma_{F e} G S_{\mathrm{o}} V_{\mathrm{o}}}{n_{\mathrm{o}}^{2} V_{\mathrm{o}}^{2}} J_{\mathrm{p}} f}-1\right) \quad\left[\mathrm{J} / \mathrm{m}^{3}\right]
$$
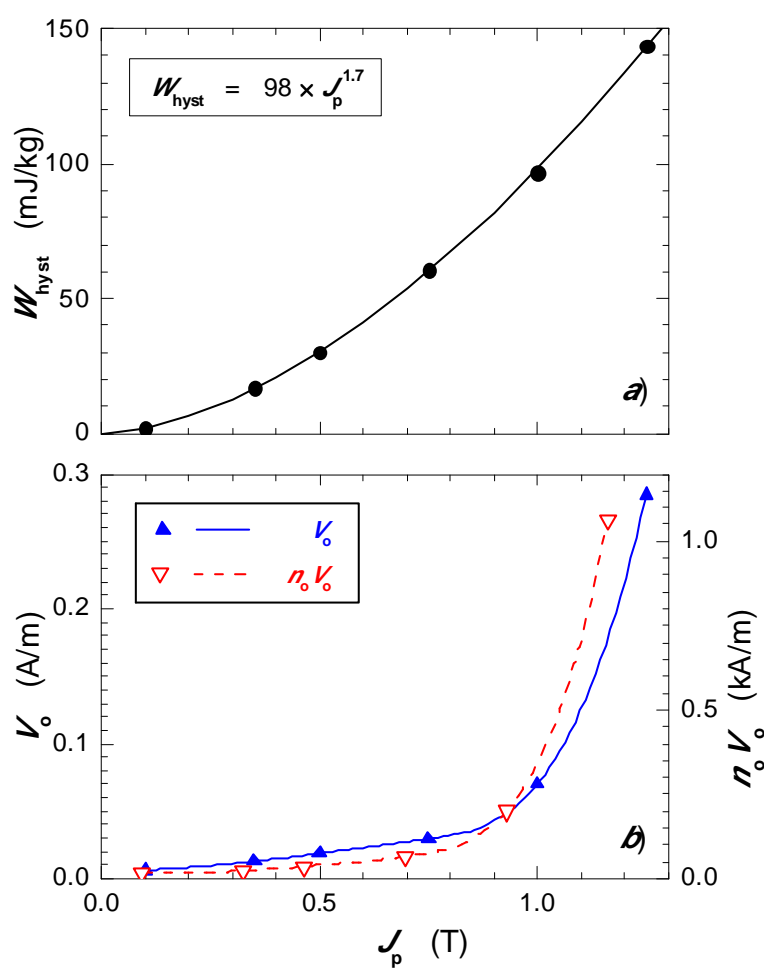

Fig. 9 - Type A Soft Magnetic Composite under sinusoidal flux. a) Hysteresis loss $W_{\text {hyst }}$ as a function of peak polarization $J_{\mathrm{p}}$. b) Parameters $V_{o}$ and $n_{o} \cdot V_{o}$ (see (7)) versus $J_{p}$.

It is fair to state that inevitable interwinding capacitive effects may hinder to some extent the achievement of a perfectly triangular $J(t)$ waveform (i.e. square voltage), because oscillations can arise at the tip points. For this reason, our experiments were performed up to the maximum exciting frequency $f=5 \mathrm{kHz}$. Fig. 13 shows the frequency dependence of the energy loss measured under triangular induction up to 5 $\mathrm{kHz}$ for $J_{\mathrm{p}}=0.5 \mathrm{~T}, 1 \mathrm{~T}$, and $1.25 \mathrm{~T}$ in the A-type material. The results show excellent agreement with the theoretical prediction. Weak effects related to the appearance of macroscopic eddy current patterns may in general be envisaged at the highest frequencies and with the strongest distortions, so the total loss can be slightly underestimated by the present theory, but in any case remains within the range of measurement uncertainty.

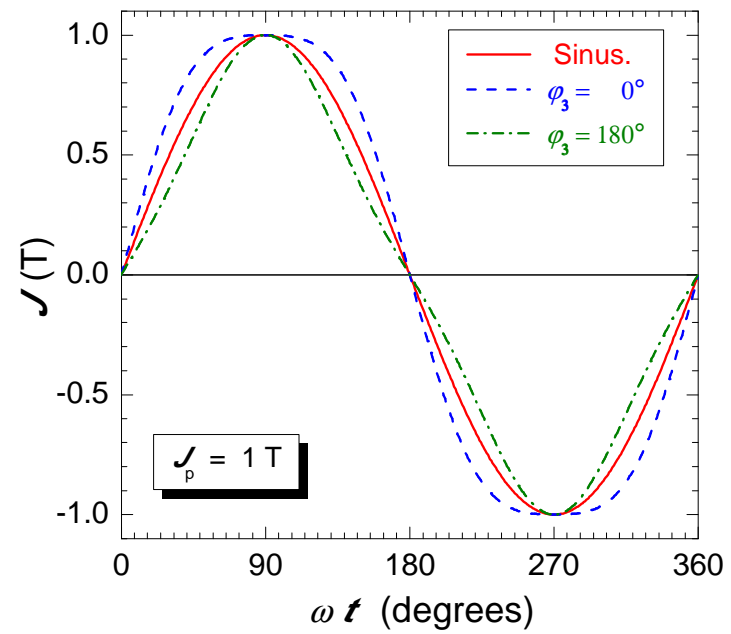

Fig. 10 - Experimental $J(t)$ waveforms obtained, according to (9), by combination of fundamental $J_{1}$ plus third $J_{3}$ harmonics. The harmonics amplitudes are in the ratio $J_{3} / J_{1}=0.1$ and $J_{\mathrm{p}}=1 \mathrm{~T}$.

\section{CONCLUSIONS}

We have carried out a comprehensive investigation on the magnetic losses in Soft Magnetic Composites from DC to 10 $\mathrm{kHz}$ and provided an associated theoretical assessment. This is based, on the one hand, on the modeling of the classical eddy current losses in heterogeneous materials and, on the other hand, on the statistical theory of losses, by which the energy loss behavior under non-sinusoidal induction waveform can be predicted. It is emphasized that eddy currents come about on two different scales, that of the individual particle (microscopic) and that of the sample cross-section (macroscopic). The latter follows from the presence of random intergrain contacts, which require sufficiently large crosssectional area to result in closed paths. Consequently, no macroscopic currents are established in sufficiently small samples (e.g. $5 \mathrm{~mm} \times 5 \mathrm{~mm}$ cross-sectional area and lower), as demonstrated by the experiments. Classical eddy current losses have been calculated for such kind of specimens, taking into account size and shape of the iron particles and the statistical distribution of their geometrical parameters.

Exploiting our ability to compute the classical loss component, we were able to make a full assessment of the magnetic loss behavior of the composites in the frequency range DC $-10 \mathrm{kHz}$ when subjected, according to standard rules, to sinusoidal induction. We were able, in particular, extract a couple of structure related parameters $\left(n_{\mathrm{o}}\left(J_{\mathrm{p}}\right)\right.$ and $\left.V_{\mathrm{o}}\left(J_{\mathrm{p}}\right)\right)$, characterizing the statistical features of the magnetization process, and consequently predict, in the framework of the 

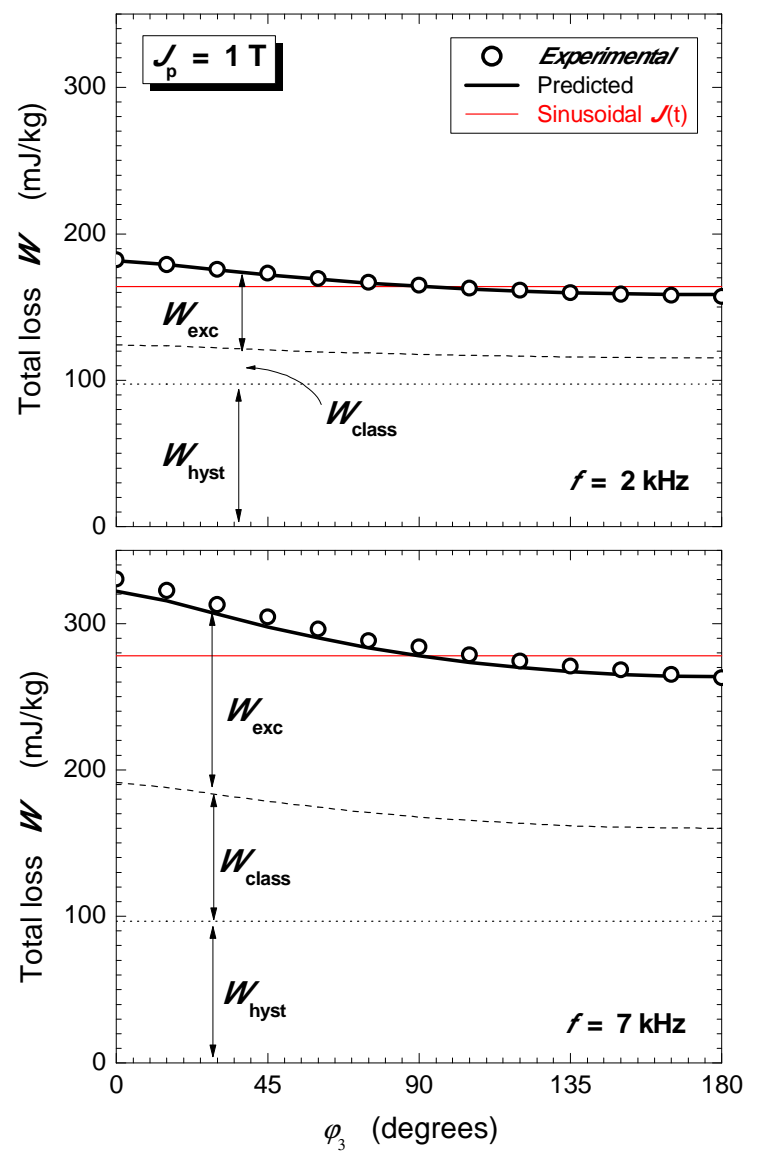

Fig. 11 - Type A Soft Magnetic Composite under non-sinusoidal flux, with distortion introduced by a third harmonic $\left(J_{\mathrm{p}}=1 \mathrm{~T}, J_{3} / J_{1}=0.1\right)$. The symbols and the solid line describe the experimental and predicted behaviors of the energy loss versus phase shift $\varphi_{3}$, respectively. The two examples reported here refer to the fundamental frequencies $f=2 \mathrm{kHz}$ and $f=7 \mathrm{kHz}$.

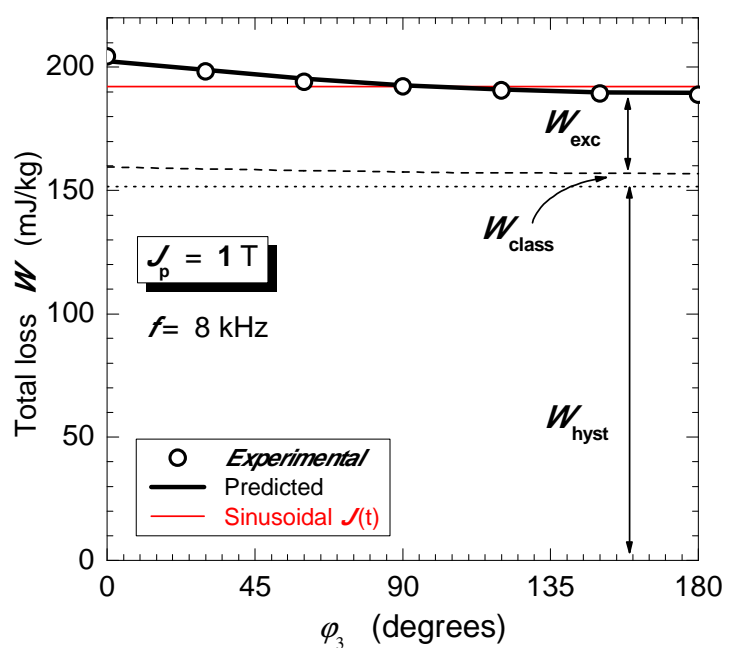

Fig. 12 - Same as Fig. 11 for the Type B Soft Magnetic Composite. Experiments (symbols) and theory (solid line) at the fundamental frequency $f=8 \mathrm{kHz}$.

loss separation principle, the effect of distortion of magnetic induction on the SMC loss behavior. Although more complex than in the conventional case of soft magnetic laminations, an analytical formulation for the magnetic losses under generic induction waveform is obtained. This implies that also the reverse is possible, where the material response to sinusoidal induction can be induced from measurements done with distorted induction. With the introduction of hysteresis loop modeling, the present treatment can be generalized to induction distortion with minor loops.
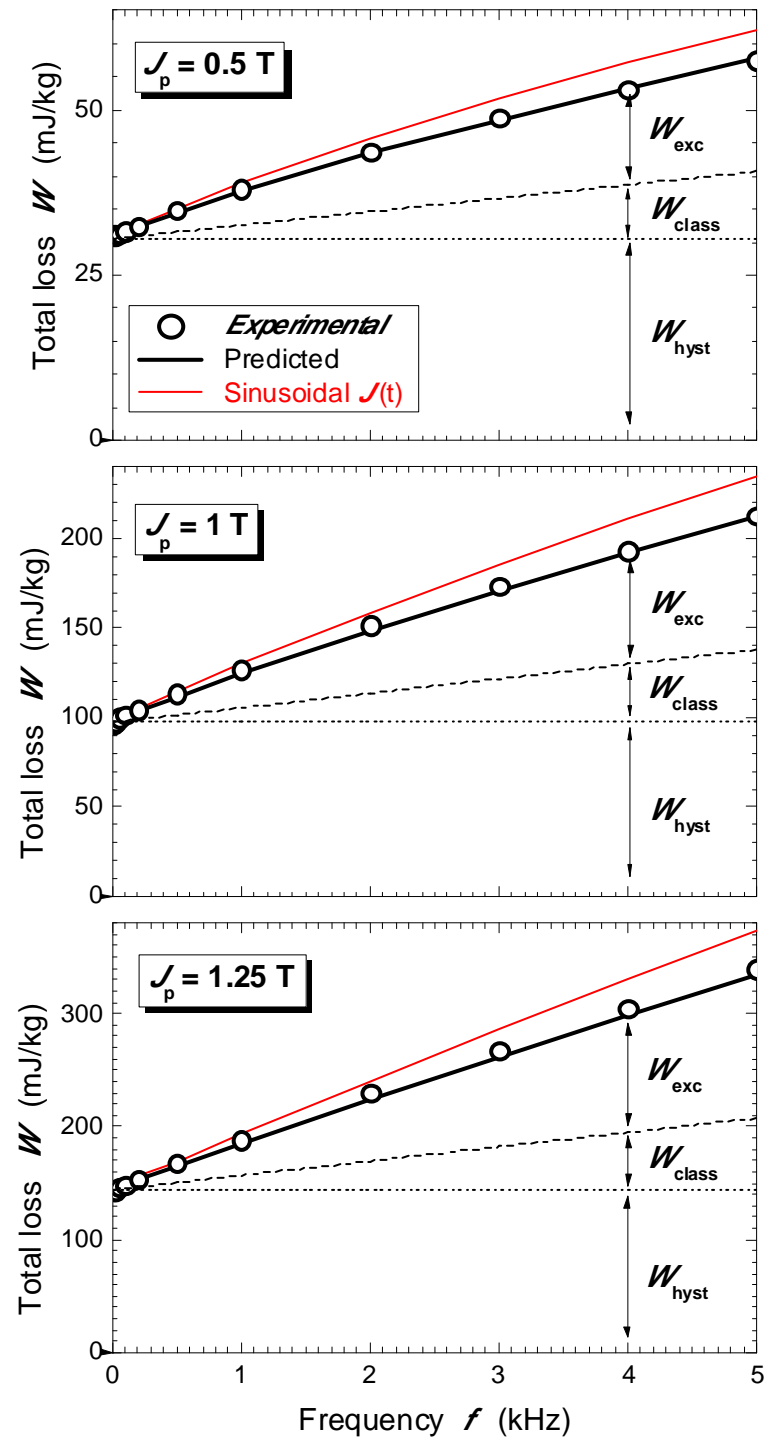

Fig. 13 - Type A Soft Magnetic Composite under triangular induction $\left(J_{\mathrm{p}}=\right.$ $0.5 \mathrm{~T}, 1.0 \mathrm{~T}, 1.25 \mathrm{~T}$ ). The experimental loss versus frequency behavior (symbols) closely compares with the prediction (solid line).

\section{APPENDIX}

In this appendix, it is demonstrated that the formula (3) tends to the one of the classical loss in the lamination, in the limit $R \rightarrow 0$ (i.e. $L>>l$ ), which should be, for a given width $l$ :

$$
W_{\text {class }}^{R \rightarrow 0}\left(J_{\mathrm{p}}, f\right)=\frac{1}{\rho_{\mathrm{Fe}}} \cdot \frac{1}{12} \cdot l^{2} \cdot\left\langle\dot{J}^{2}(t)\right\rangle \quad, \quad\left[\mathrm{J} / \mathrm{m}^{3}\right]
$$

If formula (3) is in good agreement with formula (11), recalling that $S=L . l$ and $R_{\mathrm{l}}=l / L$, one should find: 


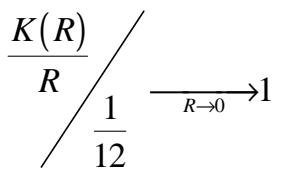

This can be verified graphically in Fig. 14. Thus the two formulae (3) and (11) are consistent.

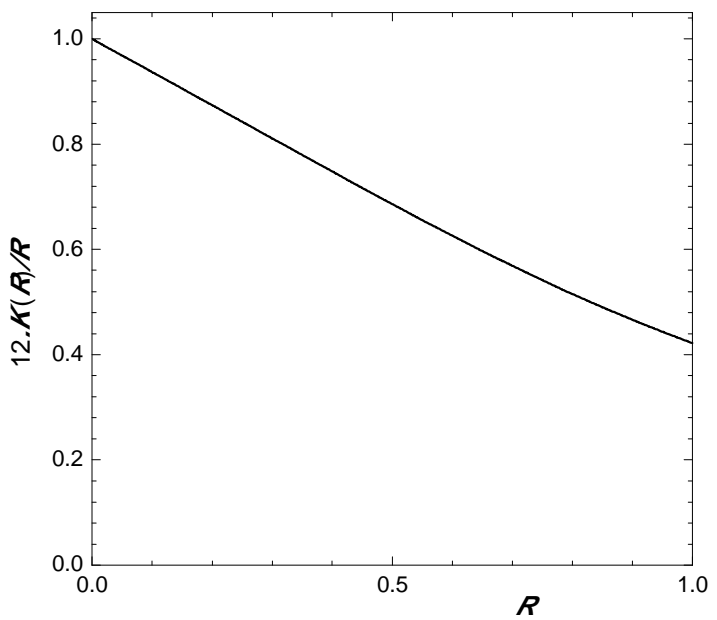

Fig. 14 - Ratio $12 \cdot K(R) / R$ to check the consistency of the two formulae (3) and (11).

\section{REFERENCES}

[1] J. Wang, T. Ibrahim, and D. Howe, "Prediction and Measurement of Iron Loss in a Short-Stroke, Single-Phase, Tubular Permanent Magnet Machine," IEEE Trans. Magn., vol. 46, no. 6, pp. 1315-1318, 2010.

[2] A. Chebak, P. Viarouge, and J. Cros, "Analytical Computation of the Full Load Magnetic Losses in the Soft Magnetic Composite Stator of High-Speed Slotless Permanent Magnet Machines," IEEE Trans. Magn., vol. 45, no. 3, pp. 952-955, 2009.

[3] W. Fei and P.C.K. Luk, "An improved model for the back-EMF and cogging torque characteristics of a novel axial flux permanent magnet synchronous machine with a segmental laminated stator," IEEE Trans. Magn., vol. 45, no. 10, pp. 4609-4612, 2009.

[4] O. Bottauscio, A. Canova, M. Chiampi, and M. Repetto, "Iron losses in electrical machines: influence of different material models," IEEE Trans. Magn., vol. 38, no. 2, pp. 805-808, 2002.

[5] K. Venkatachalam, C.R. Sullivan, T. Abdallah, and H. Tacca, "Accurate prediction of ferrite core loss with nonsinusoidal waveforms using only Steinmetz parameters," in Proc. IEEE Workshop on Computers in Power Electronics, 2002, pp. 36-41.

[6] H. Yoon, I. Kim, P.S. Shin, C.S. Koh, "Finite Element Implementation of a Generalized Chua-Type Vector Hysteresis Model and Application to Iron Loss Analysis of Three-Phase Transformer", IEEE Trans. Magn., vol. 47, no. 5, pp. 1122-1125, 2011.

[7] O. de la Barrière, C. Appino, F. Fiorillo, C. Ragusa, H. Ben Ahmed, M. Gabsi, F. Mazaleyrat, and M. LoBue, "Loss separation in soft magnetic composites," J. Appl. Phys., vol. 109, p. 07A317, 2011.

[8] G. Bertotti, "General properties of power losses in soft ferromagnetic materials," IEEE Trans. Magn., vol. 24, no. 1, pp. 621-630, 1988.

[9] F. Fiorillo and A. Novikov, "An improved approach to power losses in magnetic laminations under nonsinusoidal induction waveform", IEEE Trans. Magn., vol. 26, pp. 2904-2910, 1990.

[10] E. Barbisio, F. Fiorillo, and C. Ragusa, "Predicting Loss in Magnetic Steels Under Arbitrary Induction Waveform and With Minor Hysteresis Loops," IEEE Trans. Magn., vol. 40, no. 4, pp. 1810-1819, 2004.

[11] R. Kaczmarek, M. Amar, and F. Protat, "Iron Loss Under PWM Voltage Supply on Epstein Frame and in Induction Motor Core," IEEE Trans. Magn., vol. 32, no. 1, pp. 189-194, 1996.

[12] P. Marketos, J.P. Hall, and S.E. Zirka, "Power Loss Measurement and Prediction of Soft Magnetic Powder Composites Magnetized Under
Sinusoidal and Nonsinusoidal Excitation," IEEE Trans. Magn., vol. 44, no. 11 , pp. 3847-3850, 2008

[13] M. Anhalt and B Weidenfeller, "Dynamic losses in FeSi filled polymer bonded soft magnetic composites," Journal of Magnetism and Magnetic Materials, vol. 304, no. 2, pp. e549-e551, 2006.

[14] H. Skarrie, Design of powder core inductors, Ph.D. dissertation, University of Lund, Sweden, 2001.

[15] D. Miljavec, B. Zidaric, M. Zagirnyak, "Soft magnetic composite in design of BLDC motor", Proceedings of the International Conference on Electrical Machines (ICEM), 2004.

[16] C. Yanhong, G. Kliman, "Modeling of soft magnetic composites", Proceedings of the $39^{\text {th }}$ IEEE Industry Applications Conference (IAS), pp. 551-556, 2004.

[17] P. Kollára, Z. Bircákováa, J. Füzera, J. Füzerováb, R. Burešc, M. Fáberová, "Wide Frequency Range AC Magnetic Properties of $\mathrm{Fe}$ Based Composite Materials", Acta Physica Polonica A., vol. 118, no. 5, pp. 759-761, 2010.

[18] L. Lefebvre, S. Pelletier, C. Gélinas, "Effect of electrical resistivity on core losses in soft magnetic iron powder materials", Journal of Magnetism and Magnetic Materials, vol. 176, no. 2-3, pp. L93-L96, 1997.

[19] M. De Wulf, L. Anestiev, L. Dupré, L. Froyen, and J. Melkebeek, "Loss separation in soft magnetic composites", J. Appl. Phys., vol. 91, pp. 7845-7847, 2002.

[20] G. Nord, L. Pennander, A. Jack, "Loss calculations for soft magnetic composites", Proceedings of the International Conference on Electrical Machines (ICEM), 2004

[21] Höganäs SMC Brochures. [Online]. http://hoganas.com/en/Products-Applications/Soft-Magnetic-Composites/SMC-Brochures-Pics/

[22] O. Bottauscio, A. Manzin, V. Chiadò Piat, M. Codegone, and M. Chiampi, "Electromagnetic phenomena in heterogeneous media: effective properties and local behavior", J. Appl. Phys., vol. 100, p. 044902, 2006.

[23] S.M. Ross, Introduction to probability and statistics for engineers and scientists, Elsevier Academic Press, 2009. 